\title{
Assessment of Molecular Mechanisms and Potential Biomarkers in Bladder Urothelial Carcinoma
}

Ceren Sucularli, [PhD]

ORCID:0000-0003-0627-7900

Department of Bioinformatics, Graduate School of Health Sciences, Hacettepe University.

06100, Ankara, TURKEY

e-mail: ceren.sucularli@hacettepe.edu.tr

Tel: +903123053038

Fax: +903123051459

\section{wee) ABSTRACT cen}

Objective: Bladder cancer ranks $10^{\text {th }}$ among the most common cancers worldwide, effecting mostly man than women. The aim of this study is to perform a detailed gene expression analysis of bladder urothelial carcinoma to reveal altered molecular mechanisms and to find potential biomarkers for this cancer.

Materials and Methods: Bladder urothelial carcinoma RNA-seq data from TCGA with normal bladder samples from GTEx were analyzed by using GEPIA. Differentially expressed genes were annotated to GO-BP and KEGG pathway terms with DAVID and PPI networks were constructed by STRING. The association of upregulated cell cycle pathway proteins and patient survival was further investigated.

Results: Upregulated genes mainly annotated to cell cycle, p53 signaling and oocyte meiosis and maturation pathways and cell cycle related GO-BP terms.

Downregulated genes mostly annotated to adhesion, ECM-receptor interaction, vascular smooth muscle contraction and cardiomyopathy related KEGG pathways and muscle related GO-BP terms. The protein products of six cell cycle genes, which were upregulated in bladder urothelial carcinoma, showed significant association with patient survival.

Conclusion: The results of this study showed altered molecular mechanisms and increased our understanding of bladder urothelial carcinoma, proposed potential prognostic biomarkers.

Keywords: Prediction, protein-protein interaction networks, pathway analysis, gene expression, patient survival

Received: 12 April 2019, Accepted: 23 May 2019,

Published online: 30 June 2019

\section{INTRODUCTION}

Bladder cancer ranks $10^{\text {th }}$ among the most common cancer forms worldwide, with an anticipated 549,000 new cases and 200,000 deaths in 2018 [1]. Bladder cancer is more prevalent in man than women. It is the 6th most common cancer and ranks 9th in cancer death causes in man [1]. Bladder cancer has adaptable metastatic potential and bones, liver, peritoneum, lung and lymph nodes are the common metastasis sites of bladder cancer [2]. Genetic predisposition has been proposed to have a significant effect on bladder cancer incidence [3]. Genetic mutations of several cell division and cell cycle genes, such as HRAS, TP53, CCND1 and RB1, have been associated with bladder cancer [4]. Although exploring underlying genetic mutations are important in bladder cancer, it is also essential to reveal gene expression alterations and molecular mechanisms, especially proliferation, metastasis and cell cycle mechanisms, for facilitating diagnosis and treatment approaches in bladder cancer.

Re-analysis of gene expression datasets is a powerful tool to search altered mechanisms of cancer. Previously, studies were performed with re-analysis of microarray datasets from GEO database of 
tumor and control samples to reveal altered gene expression mechanisms of bladder urothelial carcinoma $[5,6]$. The increased expression of genes in bladder cancer found to be annotated to negative regulation apoptotic process, salmonella infection, amoebiasis, malaria pathways and rheumatoid arthritis pathways, while downregulated genes were annotated to oxidative phosphorylation, taurine and hypotaurine metabolism, vitamin digestion and absorption, arthritis and vibrio cholerae infection pathways [7]. Enrichment of upregulated genes in cell division and protein binding, enrichment of downregulated genes in extracellular matrix organization and complement and coagulation cascades was also reported [5].

Studies with TCGA datasets of bladder cancer mainly focus on genomic analysis of DNA alterations, including variations and mutations, and related pathways [8-11]. According to DNA alteration data, several critical pathways, including cell cycle pathway, have been shown to be dysregulated in bladder cancer $[8,10]$. At the gene expression level TCGA datasets were mainly used to identify molecular subtypes of bladder cancer or finding important miRNA or IncRNA signatures [8, 10, 12, 13]. Therefore, studies using TCGA RNA-seq data of bladder cancer to search for significant mRNA expression alterations and molecular mechanisms will contribute to bladder cancer research.

This study presents a detailed RNA-seq gene expression analysis with high number of bladder urothelial carcinoma and normal samples. Gene ontology (GO) terms for biological process (BP), Kyoto Encyclopedia of Genes and Genomes (KEGG) pathway analyses and protein-protein interaction network constructions were performed. The effect of gene expression alterations of selected gene products on patient survival was also investigated.

\section{MATERIALS and METHODS}

RNA-seqAnalysis of BladderUrothelial Carcinoma Bladder urothelial carcinoma RNA-seq data included 404 tumor samples and 19 normal samples from TCGA (The Cancer Genome Atlas) and 9 normal bladder samples from GTEx (Genotype-Tissue Expression). RNA-seq data was analyzed by using GEPIA (http://gepia.cancer-pku.cn/, [14]). ANOVA was applied to identify differentially expressed genes between tumor and normal samples. The Benjamini and Hochberg false discovery rate method was applied for adjusting $\mathrm{p}$-value to calculate multiple testing adjusted q-value. $|\log 2 \mathrm{FC}|=2$ and q-value $=0.001$ were designated as cutoffs to obtain differentially expressed genes.

\section{G0-BP and KEGG Pathway Analyses of Differentially Expressed Genes}

GO-BP and KEGG pathway analyses were performed by using Database for Annotation Visualization and Integrated Discovery version 6.7 (DAVID; https://david.ncifcrf.gov/, [15, 16]). Benjamini-Hochberg corrected $p$-value $<0.05$ was considered as significant.

\section{Construction of Protein-Protein Interaction (PPI) Network}

STRING version 11.0 (http://string-db.org/, [17]) was used to evaluate PPI information. Differentially expressed upregulated and downregulated genes were loaded to STRING, respectively. For network construction minimum required interaction score was set to highest confidence: 0.900 .

\section{Survival Analysis}

The cancer patient survival analysis for selected genes was evaluated with Kaplan-Meier plotter tool (http://kmplot.com/analysis/index.php, [18]). Patient cohorts, divided in two groups according to quantile expressions, were compared and hazard ratio with $95 \%$ confidence intervals and log rank $p$-value were calculated and provided with a survival curve for overall survival. The log rank $p$-value $<0.05$ considered as significant.

\section{RESULTS}

\section{Differentially Expressed Genes for BLCA}

RNA-seq expression data for bladder urothelial carcinoma $(n=404)$ was compared with related normal samples $(n=28)$ to obtain differentially expressed genes. According to this comparison, 730 genes were found to be differentially expressed between tumor and normal samples. Of the 730 genes, 171 genes were found to be upregulated, while 559 genes were downregulated.

\section{Functional Annotation of Differentially Expressed Genes}

To identify molecular mechanisms that were altered in bladder urothelial carcinoma, upregulated and downregulated differentially expressed genes were annotated to GO-BP terms and KEGG pathway terms. Upregulated genes mainly annotated to cell 
Table 1. The KEGG pathway annotations and top 20 GO-BP terms of differentially expressed upregulated genes in bladder urothelial carcinoma (Benjamini<0.05).

\begin{tabular}{|c|c|c|c|}
\hline KEGG pathway term & Count & P-value & Benjamini \\
\hline hsa04110:Cell cycle & 18 & $1.34 \mathrm{E}-16$ & $4.88 \mathrm{E}-15$ \\
\hline hsa04114:Oocyte meiosis & 11 & $3.16 \mathrm{E}-08$ & $6.95 \mathrm{E}-07$ \\
\hline hsa04914:Progesterone-mediated oocyte maturation & 8 & $9.71 \mathrm{E}-06$ & $1.42 \mathrm{E}-04$ \\
\hline hsa04115:p53 signaling pathway & 6 & 3.30E-04 & 3.63E-03 \\
\hline GO-BP term & Count & P-value & Benjamini \\
\hline GO:0022403 cell cycle phase & 60 & 4.47E-59 & $3.94 \mathrm{E}-56$ \\
\hline GO:0000279 M phase & 56 & $1.27 \mathrm{E}-58$ & $5.58 \mathrm{E}-56$ \\
\hline GO:0007049 cell cycle & 68 & $2.02 \mathrm{E}-53$ & $5.93 E-51$ \\
\hline GO:0022402 cell cycle process & 61 & $2.64 \mathrm{E}-52$ & $5.82 \mathrm{E}-50$ \\
\hline GO:0000278 mitotic cell cycle & 53 & $5.47 \mathrm{E}-51$ & $9.64 \mathrm{E}-49$ \\
\hline GO:0000280 nuclear division & 45 & $1.06 \mathrm{E}-49$ & $1.55 \mathrm{E}-47$ \\
\hline GO:0007067 mitosis & 45 & $1.06 \mathrm{E}-49$ & $1.55 \mathrm{E}-47$ \\
\hline GO:0000087 M phase of mitotic cell cycle & 45 & $2.50 \mathrm{E}-49$ & $3.14 \mathrm{E}-47$ \\
\hline GO:0048285 organelle fission & 45 & 7.14E-49 & $7.86 \mathrm{E}-47$ \\
\hline GO:0051301 cell division & 41 & $7.98 \mathrm{E}-38$ & $7.81 \mathrm{E}-36$ \\
\hline GO:0007059 chromosome segregation & 20 & $9.32 \mathrm{E}-23$ & $8.21 \mathrm{E}-21$ \\
\hline GO:0007017 microtubule-based process & 26 & $5.13 \mathrm{E}-20$ & $4.11 \mathrm{E}-18$ \\
\hline GO:0007051 spindle organization & 15 & $6.01 \mathrm{E}-19$ & $4.41 \mathrm{E}-17$ \\
\hline GO:0051726 regulation of cell cycle & 27 & $2.71 \mathrm{E}-18$ & $1.84 \mathrm{E}-16$ \\
\hline GO:0000226 microtubule cytoskeleton organization Organization & 19 & $2.85 \mathrm{E}-16$ & $2.10 \mathrm{E}-14$ \\
\hline GO:0007346 regulation of mitotic cell cycle & 19 & $5.20 \mathrm{E}-16$ & $3.26 \mathrm{E}-14$ \\
\hline GO:0000070 mitotic sister chromatid segregation Segregation & 11 & $2.80 \mathrm{E}-13$ & $1.54 \mathrm{E}-11$ \\
\hline GO:0000819 sister chromatid segregation & 11 & $3.80 \mathrm{E}-13$ & 1.97E-11 \\
\hline GO:0010564 regulation of cell cycle process & 14 & $1.28 \mathrm{E}-11$ & $6.27 \mathrm{E}-10$ \\
\hline GO:0000075 cell cycle checkpoint & 13 & $1.43 \mathrm{E}-11$ & 6.61E-10 \\
\hline
\end{tabular}

KEGG, Kyoto Encyclopedia of Genes and Genomes; GO-BP, Gene Ontology-Biological Process; Count, no. of genes in term; p-value, modified Fisher exact p-value, EASE score; Benjamini, Benjamini-Hochberg corrected p-value.

Downregulated genes in bladder urothelial carcinoma cells mostly annotated to adhesion, ECM-receptor interaction, vascular smooth muscle contraction and cardiomyopathy related KEGG pathways (Table 2), muscle related GO$\mathrm{BP}$ terms, such as muscle organ development, construction and system process, biological adhesion, and circulatory system related GO-BP terms (Table 2). 
Table 2. The KEGG pathway annotations and top 20 GO-BP terms of differentially expressed downregulated genes in bladder urothelial carcinoma (Benjamini<0.05).

\begin{tabular}{|c|c|c|c|}
\hline KEGG pathway term & Count & P-value & Benjamini \\
\hline hsa04270:Vascular smooth muscle contraction & 21 & $1.05 \mathrm{E}-09$ & $1.33 \mathrm{E}-07$ \\
\hline hsa05414:Dilated cardiomyopathy & 16 & 4.99E-07 & $3.17 \mathrm{E}-05$ \\
\hline hsa04020:Calcium signaling pathway & 22 & $6.01 \mathrm{E}-07$ & $2.54 \mathrm{E}-05$ \\
\hline hsa05410:Hypertrophic cardiomyopathy (HCM) & 14 & $6.23 \mathrm{E}-06$ & $1.98 \mathrm{E}-04$ \\
\hline hsa04610:Complement and coagulation cascades & 12 & $2.21 \mathrm{E}-05$ & $5.60 \mathrm{E}-04$ \\
\hline hsa04510:Focal adhesion & 18 & $5.90 \mathrm{E}-04$ & $1.24 \mathrm{E}-02$ \\
\hline hsa04512:ECM-receptor interaction & 10 & 2.47E-03 & 4.39E-02 \\
\hline GO-BP term & Count & P-value & Benjamini \\
\hline GO:0007517 muscle organ development & 30 & $3.16 \mathrm{E}-12$ & 7.05E-09 \\
\hline GO:0007155 cell adhesion & 56 & $1.38 \mathrm{E}-11$ & $1.54 \mathrm{E}-08$ \\
\hline GO:0022610 biological adhesion & 56 & $1.45 \mathrm{E}-11$ & $1.08 \mathrm{E}-08$ \\
\hline GO:0030198 extracellular matrix organization & 20 & $1.34 \mathrm{E}-10$ & 7.47E-08 \\
\hline GO:0006936 muscle contraction & 23 & $5.82 \mathrm{E}-10$ & $2.60 \mathrm{E}-07$ \\
\hline GO:0003012 muscle system process & 24 & $6.36 \mathrm{E}-10$ & $2.37 \mathrm{E}-07$ \\
\hline GO:0043062 extracellular structure organization & 23 & $1.99 \mathrm{E}-09$ & $6.34 \mathrm{E}-07$ \\
\hline GO:0010033 response to organic substance & 51 & $9.18 \mathrm{E}-09$ & $2.56 \mathrm{E}-06$ \\
\hline GO:0044057 regulation of system process & 30 & $2.85 \mathrm{E}-08$ & 7.07E-06 \\
\hline GO:0006875 cellular metal ion homeostasis & 23 & $6.17 \mathrm{E}-08$ & $1.38 \mathrm{E}-05$ \\
\hline GO:0003018 vascular process in circulatory system & 13 & 8.38E-08 & $1.70 \mathrm{E}-05$ \\
\hline GO:0009611 response to wounding & 40 & $9.53 \mathrm{E}-08$ & 1.77E-05 \\
\hline GO:0035295 tube development & 24 & $1.15 \mathrm{E}-07$ & $1.98 \mathrm{E}-05$ \\
\hline GO:0055065 metal ion homeostasis & 23 & $1.37 \mathrm{E}-07$ & $2.19 \mathrm{E}-05$ \\
\hline GO:0042127 regulation of cell proliferation & 51 & $1.50 \mathrm{E}-07$ & $2.24 \mathrm{E}-05$ \\
\hline GO:0009719 response to endogenous stimulus & 33 & $3.03 \mathrm{E}-07$ & 4.23E-05 \\
\hline GO:0048878 chemical homeostasis & 38 & 3.32E-07 & 4.35E-05 \\
\hline GO:0050801 ion homeostasis & 33 & $3.79 \mathrm{E}-07$ & 4.70E-05 \\
\hline GO:0008015 blood circulation & 21 & 4.97E-07 & $5.83 \mathrm{E}-05$ \\
\hline GO:0003013 circulatory system process & 21 & 4.97E-07 & $5.83 \mathrm{E}-05$ \\
\hline
\end{tabular}

KEGG, Kyoto Encyclopedia of Genes and Genomes; GO-BP, Gene Ontology-Biological Process; Count, no. of genes in term; p-value, modified Fisher exact p-value, EASE score; Benjamini, Benjamini-Hochberg corrected p-value. 


\section{PPI Networks for Differentially Expressed Genes}

PPI networks of differentially expressed genes were constructed using STRING version 11.0. This PPI networks for upregulated genes consisted of 147 nodes and 1351 edges, while downregulated genes consisted of 524 nodes and 453 edges. Average node degree for upregulated genes was 18.4 , while it was 1.73 for downregulated genes.

STRING database provided information on gene annotations to pathway terms (Table 3). The protein products of upregulated genes that functioned in cell cycle pathway KEGG term were selected for further analysis (Figure 1).

Figure 1. PPI network of differentially upregulated genes. Red nodes showed proteins annotated in cell cycle pathway term.

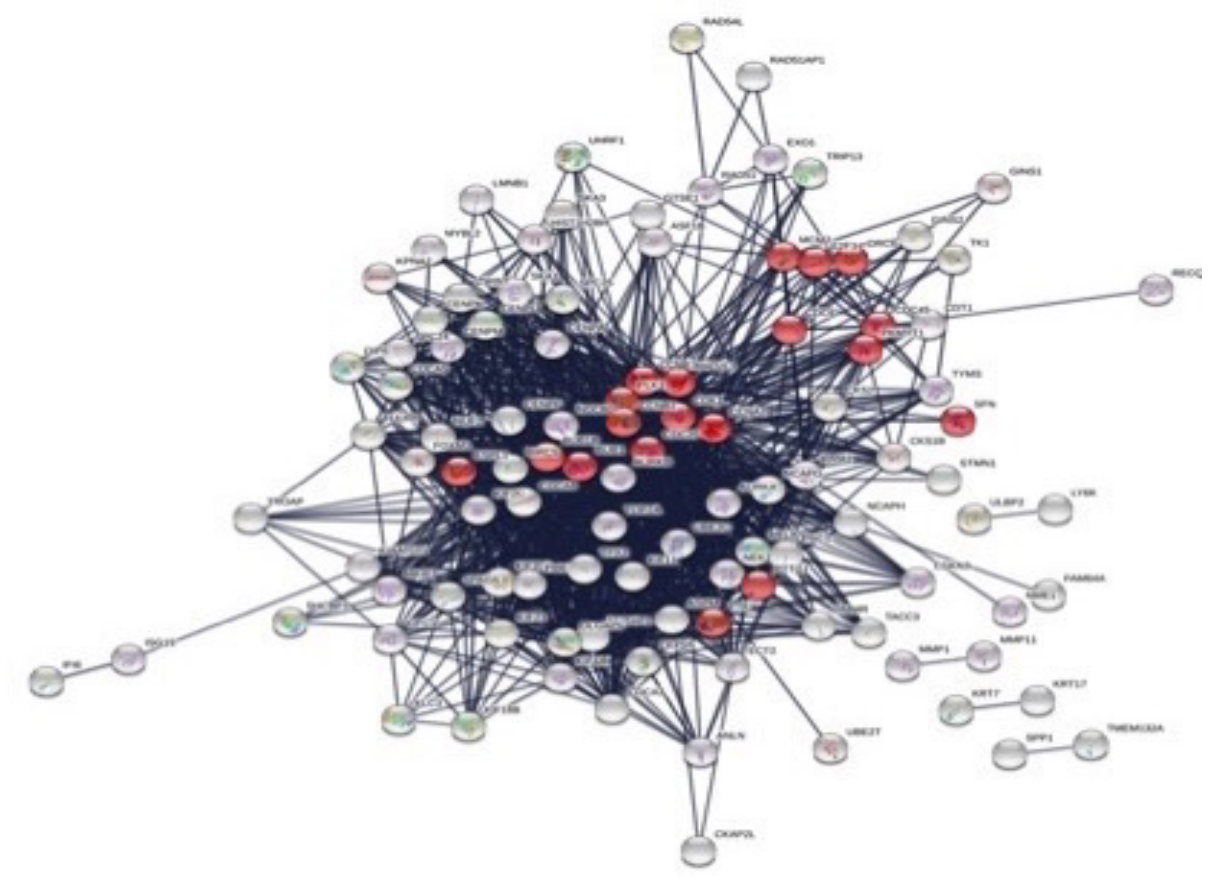

Table 3. KEGG pathway annotations of differentially expressed upregulated genes in bladder urothelial carcinoma from STRING database $(\mathrm{FDR}<0.05)$.

\begin{tabular}{|c|c|c|}
\hline KEGG pathway term & FDR & Proteins in network \\
\hline hsa04110: Cell cycle & $7.81 \mathrm{E}-17$ & $\begin{array}{c}\text { BUB1, BUB1B, CCNA2, CCNB1, CCNB2, CDC20, CDC45, } \\
\text { CDC6, CDK1, E2F1, ESPL1, MAD2L1, MCM2, ORC6, PKMYT1, } \\
\text { PLK1, PTTG1, SFN, TTK }\end{array}$ \\
\hline hsa04114: Oocyte meiosis & 1.17E-07 & $\begin{array}{c}\text { AURKA, BUB1, CCNB1, CCNB2, CDC20, CDK1, ESPL1, } \\
\text { MAD2L1, PKMYT1, PLK1, PTTG1 }\end{array}$ \\
\hline $\begin{array}{l}\text { hsa04914: Progesterone-mediated oocyte } \\
\text { maturation }\end{array}$ & $2.08 \mathrm{E}-06$ & $\begin{array}{c}\text { AURKA, BUB1, CCNA2, CCNB1, CCNB2, CDK1, MAD2L1, } \\
\text { PKMYT1, PLK1 }\end{array}$ \\
\hline hsa04115: p53 signaling pathway & $3.80 \mathrm{E}-04$ & CCNB1, CCNB2, CDK1, GTSE1, RRM2, SFN \\
\hline hsa04218: Cellular senescence & $3.60 \mathrm{E}-03$ & CCNA2, CCNB1, CCNB2, CDK1, E2F1, FOXM1, MYBL2 \\
\hline hsa05166: HTLV-I infection & $4.32 \mathrm{E}-02$ & BUB1B, CCNB2, CDC20, E2F1, MAD2L1, MYBL2, PTTG1 \\
\hline
\end{tabular}




\section{Survival Analysis of Cell Cycle Proteins}

The effect of increased expression of the proteins, which functioned in cell cycle pathway, on overall patient survival was investigated to find potential prognostic biomarkers of bladder urothelial carcinoma.

18 genes were common in cell cycle KEGG pathway term in DAVID and STRING annotations, yet STRING results gave also ORC6 in KEGG pathway. The effect of total 19 proteins from DAVID and STRING annotations from cell cycle pathway on patient survival was investigated and six proteins have been found to be associated with overall survival in bladder carcinoma (Figure 2A-F).
A)

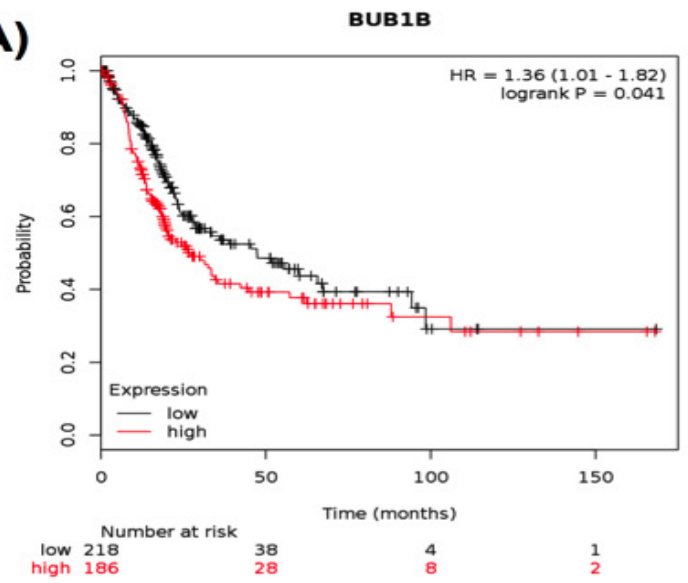

C)

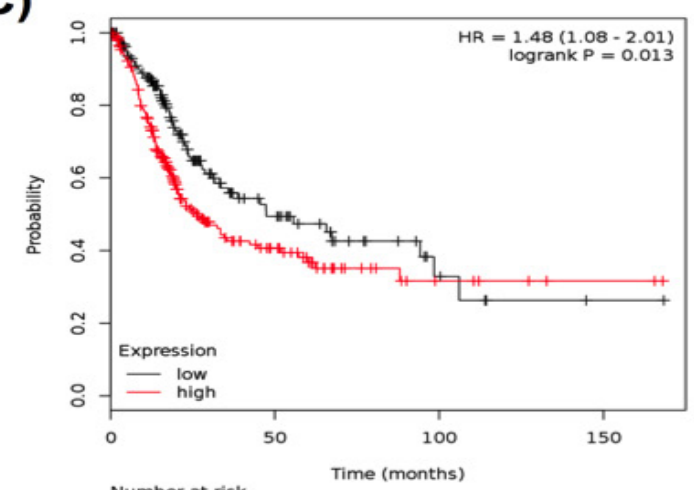

Number at risk
low 164
high 240

E)

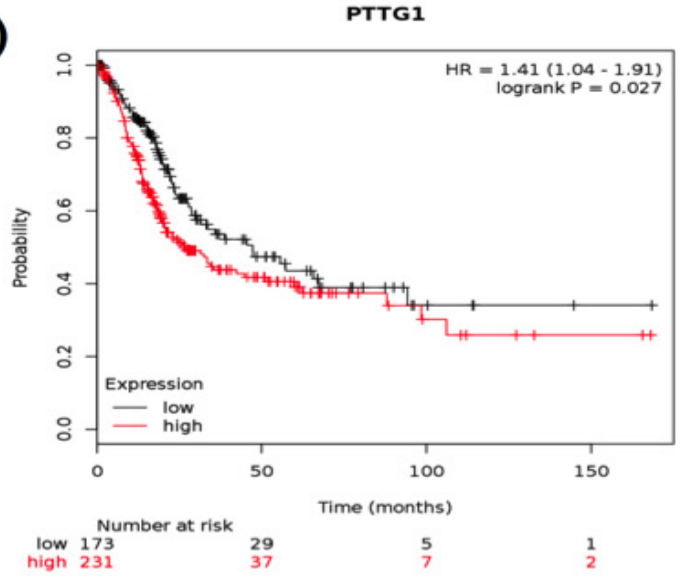

B)

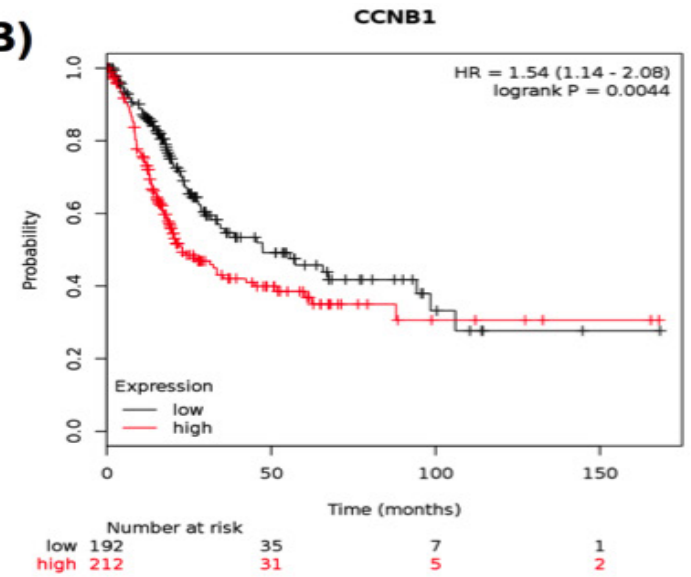

D)
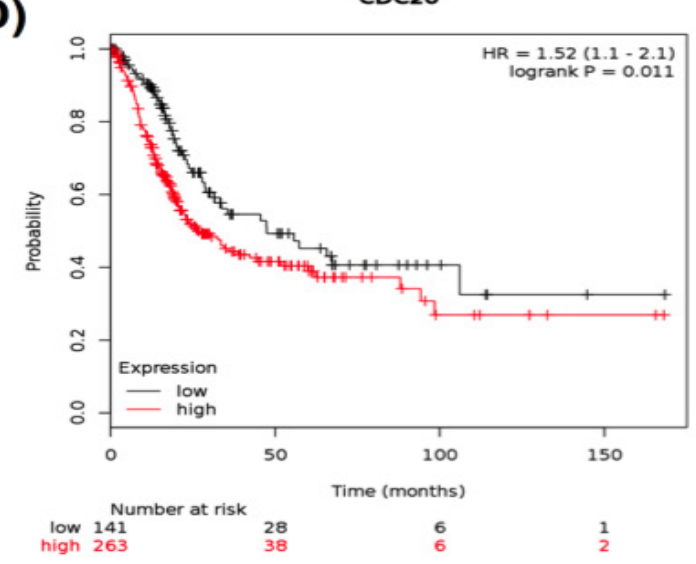

F)

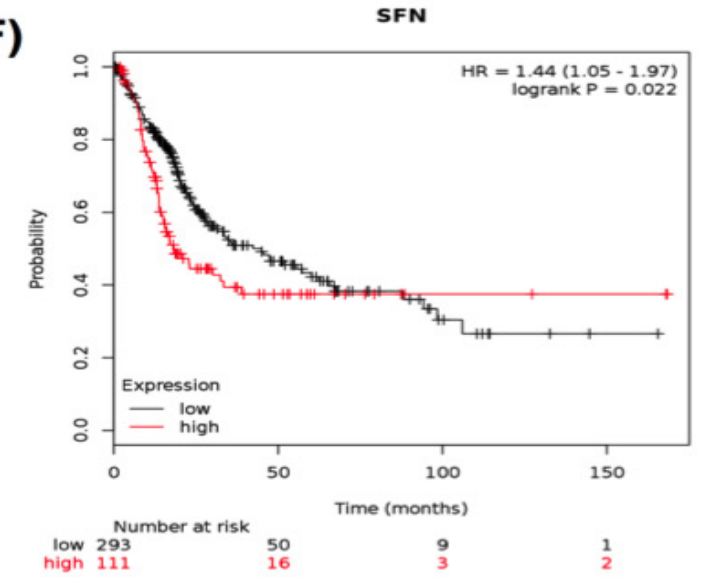

Figure 2. Kaplan-Meier survival plots showing overall survival for A) BUB1B, B) CCNB1, C) PLK1, D) CDC20, E) PTTG1 and F) SFN. 


\section{DISCUSSION}

In this study, molecular mechanisms and potential prognostic biomarkers of bladder urothelial carcinoma were investigated. RNA-seq gene expression analysis was performed with high number of bladder urothelial carcinoma and normal samples. GO-BP and KEGG pathway analyses was performed, PPI networks were constructed for differentially expressed upregulated and downregulated genes. The effect of gene expression alterations of cell cycle KEGG pathway proteins on patient survival was also explored.

Here, upregulated genes mainly annotated to cell cycle pathway and cell cycle related GO-BP terms (Table 1). Annotation of upregulated genes to cell cycle pathway and cell cycle related GO-BP terms might indicate the increased mitosis and cell division in bladder urothelial carcinoma cells. The upregulation of genes in cell division terms is consistent with previous report showing enrichment of differentially expressed upregulated genes in cell division and cell cycle related terms in bladder cancer [5]. According to KEGG pathway and GO-BP term annotations, downregulated genes in bladder urothelial carcinoma cells mostly annotated to adhesion, ECM-receptor interaction, cardiomyopathy related pathways, muscle related and circulatory system related GO-BP terms (Table 2). Alteration of ECM and cell adhesion has been shown to be important processes in tumor invasion and metastasis for several cancer types [19]. Interestingly, genes related with cardiomyophaty related pathways were found to be downregulated (Table 2). The appearance of cardiomyopathy here may be related with patient history, since different chemotherapeutics have been shown to have cardiotoxicity potential [20].

In this study, the association of increased expression of 19 genes that were annotated to cell cycle pathway with patient prognosis was investigated and high expression of 6 cell cycle pathway genes were found to be associated with bladder cancer patient prognosis (Figure 2A-F). Increased CCNB1 (cyclin B1) expression and tumor growth due to CCNB1 have been shown in cancers, such as hepatocellular carcinoma and colorectal cancer [21, 22]. Overexpression of CDC20 (cell division cycle 20) has been observed in cancers and CDC20 proposed as marker for ovarian cancer [23, 24]. CDC20 and CCNB1 were previously associated with patient prognosis in bladder cancers [25], which confirms the results presented in this study (Figure 2B and Figure 2D). Increased expression of BUB1B (BUB1 mitotic checkpoint serine/ threonine kinase $B$ ) has been shown in breast cancer and proposed as a potential marker for breast cancer classification [26]. Increased PLK1 (polo like kinase 1) and PTTG1 (PTTG1 regulator of sister chromatid separation, securin) expressions were detected in different bladder cancer cell lines and tissues [27-29]. The expression alteration of SFN (Stratifin or 14-3-3 protein sigma) has not been studied in bladder cancer in detail, although genetic variation and methylation status of SFN were studied in bladder cancer [30,31]. Here, in addition to showing increased expression of PTTG1, SFN, BUB1B and PLK1 in bladder carcinoma patients (Figure 1), expression of these proteins was shown to be associated with patient survival (Figure 2).

The results presented in this study showed the altered molecular mechanisms in bladder urothelial carcinoma patients. Moreover, the prognostic and predictive value of gene products of upregulated genes that were annotated to cell cycle pathway by DAVID and STRING was investigated and the expression of six genes were associated with patient survival.

\section{CONFLICT Of INTEREST}

There is no conflict of interest. This research did not receive any specific grant from funding agencies in the public, commercial, or not-for-profit sectors.

\section{ACKNOWLEDGEMENT}

The author is grateful to Hacettepe University, Graduate School of Health Sciences and Department of Bioinformatics for providing facilities for this study. 
[1] Bray F, Ferlay J, Soerjomataram I, et al. Global cancer statistics 2018: GLOBOCAN estimates of incidence and mortality worldwide for 36 cancers in 185 countries. CA Cancer J Clin 2018; 68: 394-424.

[2] Shinagare $A B$, Ramaiya $N H$, Jagannathan JP, et al. Metastatic pattern of bladder cancer: correlation with the characteristics of the primary tumor. AJR Am J Roentgenol 2011; 196: 117-22.

[3] Burger M, Catto JW, Dalbagni G, et al. Epidemiology and risk factors of urothelial bladder cancer. Eur Urol 2013; 63: 234-41.

[4] Zhang X, Zhang Y. Bladder Cancer and Genetic Mutations. Cell Biochem Biophys 2015; 73: 65-9.

[5] Tang F, He Z, Lei $\mathrm{H}$, et al. Identification of differentially expressed genes and biological pathways in bladder cancer. Mol Med Rep 2018; 17: 6425-34.

[6] Di Y, Chen D, Yu W, et al. Bladder cancer stage-associated hub genes revealed by WGCNA co-expression network analysis. Hereditas 2019; 156: 7.

[7] Yan $M$, Jing $X$, Liu $Y$, et al. Screening and identification of key biomarkers in bladder carcinoma: Evidence from bioinformatics analysis. Oncol Lett 2018; 16: 3092-100.

[8] Robertson AG, Kim J, Al-Ahmadie H, et al. Comprehensive Molecular Characterization of Muscle-Invasive Bladder Cancer. Cell 2017; 171: 540-56 e25.

[9] Choi W, Ochoa A, McConkey DJ, et al. Genetic Alterations in the Molecular Subtypes of Bladder Cancer: Illustration in the Cancer Genome Atlas Dataset. Eur Urol 2017; 72: 354-65.

[10] Cancer Genome Atlas Research N. Comprehensive molecular characterization of urothelial bladder carcinoma. Nature 2014; 507: 315-22.

[11] Glaser AP, Fantini D, Wang Y, et al. APOBEC-mediated mutagenesis in urothelial carcinoma is associated with improved survival, mutations in DNA damage response genes, and immune response. Oncotarget 2018; 9: 4537-48.

[12] Gao JM, Huang LZ, Huang ZG, et al. Clinical value and potential pathways of miR-183-5p in bladder cancer: A study based on miRNA-seq data and bioinformatics analysis. Oncol Lett 2018; 15: 5056-70.

[13] He RQ, Huang ZG, Li TY, et al. RNA-Sequencing Data Reveal a Prognostic Four-IncRNA-Based Risk Score for Bladder Urothelial Carcinoma: An in Silico Update. Cell Physiol Biochem 2018; 50: 1474-95.

[14] Tang Z, Li C, Kang B, et al. GEPIA: a web server for cancer and normal gene expression profiling and interactive analyses. Nucleic Acids Res 2017; 45: W98-W102.

[15] Huang da W, Sherman BT, Lempicki RA. Bioinformatics enrichment tools: paths toward the comprehensive functional analysis of large gene lists. Nucleic Acids Res 2009; 37: 1-13.

[16] Huang da W, Sherman BT, Lempicki RA. Systematic and integrative analysis of large gene lists using DAVID bioinformatics resources. Nat Protoc 2009; 4: 44-57.

[17] Szklarczyk D, Gable AL, Lyon D, et al. STRING v11: protein-protein association networks with increased coverage, supporting functional discovery in genome-wide experimental datasets. Nucleic Acids Res 2019; 47: D607-D13.

[18] Nagy A, Lanczky A, Menyhart O, et al. Validation of miRNA prognostic power in hepatocellular carcinoma using expression data of independent datasets. Sci Rep 2018; 8: 9227.

[19] Jinka R, Kapoor R, Sistla PG, et al. Alterations in CellExtracellular Matrix Interactions during Progression of Cancers. Int J Cell Biol 2012; 2012: 219196.

[20] Herrmann J, Lerman A, Sandhu NP, et al. Evaluation and management of patients with heart disease and cancer: cardio-oncology. Mayo Clin Proc 2014; 89: 1287-306.

[21] Chai N, Xie HH, Yin JP, et al. FOXM1 promotes proliferation in human hepatocellular carcinoma cells by transcriptional activation of CCNB1. Biochem Biophys Res Commun 2018; 500: 924-9.

[22] Fang $Y, Y u ~ H$, Liang $X$, et al. Chk1-induced CCNB1 overexpression promotes cell proliferation and tumor growth in human colorectal cancer. Cancer Biol Ther 2014; 15: 1268-79.

[23] Ouellet V, Guyot MC, Le Page C, et al. Tissue array analysis of expression microarray candidates identifies markers associated with tumor grade and outcome in serous epithelial ovarian cancer. Int J Cancer 2006; 119: 599-607.

[24] Wang Z, Wan L, Zhong J, et al. Cdc20: a potential novel therapeutic target for cancer treatment. Curr Pharm Des 2013; 19: 3210-4.

[25] Gao X, Chen Y, Chen M, et al. Identification of key candidate genes and biological pathways in bladder cancer. PeerJ 2018; 6: e6036.

[26] Yuan B, Xu Y, Woo JH, et al. Increased expression of mitotic checkpoint genes in breast cancer cells with chromosomal instability. Clin Cancer Res 2006; 12: 405-10.

[27] Zhang Z, Zhang G, Gao Z, et al. Comprehensive analysis of differentially expressed genes associated with PLK1 in bladder cancer. BMC Cancer 2017; 17: 861.

[28] Zhang Z, Zhang G, Kong C. High expression of polo-like kinase 1 is associated with the metastasis and recurrence in urothelial carcinoma of bladder. Urol Oncol 2013; 31: 1222-30.

[29] Xiang W, Wu X, Huang C, et al. PTTG1 regulated by miR$146 a-3 p$ promotes bladder cancer migration, invasion, metastasis and growth. Oncotarget 2017; 8: 664-78.

[30] Pineda S, Milne RL, Calle ML, et al. Genetic variation in the TP53 pathway and bladder cancer risk. a comprehensive analysis. PLoS One 2014; 9: e89952.

[31] Negraes PD, Favaro FP, Camargo JL, et al. DNA methylation patterns in bladder cancer and washing cell sediments: a perspective for tumor recurrence detection. BMC Cancer 2008; 8: 238. 